\title{
A Comparison of Item- and Person-Fit Methods of Assessing Model-Data Fit in IRT
}

\author{
Steven P. Reise \\ University of Minnesota
}

\begin{abstract}
Many item-fit statistics have been proposed for assessing whether the responses to test items aggregated across examinees conform to IRT test models. Conversely, person-fit statistics have been proposed for assessing whether an examinee's responses aggregated across items are congruent with a specified IRT model. Statistical procedures to assess item fit have differed from those to assess person fit. This research compared a $\chi^{2}$ item-fit index with a likelihood-based person-fit index. Eight 0,1 data matrices were simulated under the threeparameter logistic test model. Both the likelihoodbased and $\chi^{2}$ fit statistics were then computed for examinees and items, and Type I and Type II error rates were analyzed. With data simulated to fit the IRT model, the $\chi^{2}$ test overidentified examinees and items as being misfitting, while the likelihoodbased fit index held closer to the specified $\alpha$ levels. The two fit indices gave consistent (mis)fit-tomodel results in 94 and 97 percent of cases for items and examinees, respectively, across simulations. Under simulated conditions of data misfit, the $\chi^{2}$ statistic detected misfit at a higher rate than the likelihood-based statistic, indicating that the $\chi^{2}$ statistic was slightly more sensitive to response pattern aberrancy. However, other considerations led to a recommendation for employing the likelihoodbased index in applied fit analyses to evaluate both examinee and item model-data (mis)fit. Index terms: chi-square index, item fit, item response theory, model fit, person fit, response aberrancy.
\end{abstract}

Model-data fit issues are a major concern when applying item response theory (IRT) models to real test data. Historically, the major research concern has been with item fit (e.g., Andersen, 1973; Yen, 1981). More recently, a sizable literature has developed around the issue of assessing person fit (e.g., Levine \& Drasgow, 1982; APPLIED PSYCHOLOGICAL MEASUREMENT Vol. 14, No. 2, June 1990, pp. 127-137

(C) Copyright 1990 Applied Psychological Measurement Inc. 0146-6216/90/020127-11\$1.80
Tatsuoka \& Linn, 1983). Although item fit has taken precedence in the research literature, both types of fit analysis are important. Poor item fit indicates that the item parameters have questionable validity (i.e., they do not accurately represent how examinees respond to test items), while poor person fit indicates that the trait level $(\theta)$ estimate has questionable validity (i.e., the $\theta$ estimate may be a poor indicator of an examinee's position on the latent continuum).

A main purpose of this research was to demonstrate empirically that some methods used to assess item fit can be readily applied to assess person fit, and vice versa. To accomplish this, the performance of a $\chi^{2}$ item-fit statistic was compared with that of a likelihood-based person-fit statistic when each was calculated for both examinees and items under monte carlo conditions.

\section{The Purpose and Calculation of Item Fit}

IRT requires a formal investigation of the manner in which test items function as trait measures. Once a formal model has been defined and item response functions (IRFs) have been estimated, studies must be conducted to verify that the observed data conform to the estimated IRFs. Without item-fit analysis, the researcher cannot be confident whether the advantages accrued by specifying the formal IRT model can or will be realized.

Several key issues are involved in examining item fit:

1. Model selection has been an important motivation behind item-fit studies (Yen, 1981); it is important to identify the most efficient test model that retains the integrity of the observed data. 
2. For most IRT models to be applicable, the item pool must produce item responses that approximately meet the unidimensionality assumption. Item-fit analysis has been proposed as a method of locating extraneous dimensions affecting the responses to test items (McKinley \& Mills, 1985).

3. Item-fit studies may assist in identifying faulty item construction (e.g., incorrect item keying).

4. Item fit has the potential to indicate errors that occurred in the calibration phase of test development. For example, if a program consistently underestimates the discrimination parameter for highly discriminating items, an item-fit statistic should identify this problem. Graphical methods (Kingston \& Dorans, 1985) and statistical methods (McKinley \& Mills, 1985) have been developed for assessing item fit. Most statistical methods rely on calculating a $\chi^{2}$ statistic. To implement a $\chi^{2}$ test, examinees are rankordered according to estimated $\theta$ and then grouped into some fixed or subjectively determined number of categories. Within each grouping, the proportion of examinees responding to the item in the keyed direction is calculated. This observed proportion is then compared to the predicted proportion based on the IRF. For example, the Bock (1972) $\chi_{B}^{2}$ is given by

$\chi_{\mathbf{B}_{i}}^{2}=\sum_{j=1}^{G} \frac{N_{j}\left(O_{i j}-E_{i j}\right)^{2}}{E_{i j}\left(1-E_{\imath j}\right)}$,

where $i$ is the item,

$j$ is the interval created by grouping examinees on the basis of their $\theta$ estimates,

$G$ is the number of examinee groupings,

$N$, is the number of examinees with $\theta$ estimates falling in a given interval j,

$O_{\imath \jmath}$ is the observed proportion of keyed responses on item $i$ for interval $j$, and

$E_{l j}$ is the expected proportion of keyed responses on item $i$ for interval $j$ based on the IRF evaluated at the median $\theta$ estimate within the interval.

High values of $\chi_{\mathrm{B}}^{2}$ diagnose items that are performing differentially with respect to the IRT model (i.e., items that do not fit the model). The significance of $\chi_{B}^{2}$ is tested using the $\chi^{2}$ distribution with degrees of freedom equal to the number of groupings $(G)$ minus the number of estimated parameters.

The various $\chi^{2}$ measures (e.g., Wright \& Mead, 1977; Wright \& Panchapakesan, 1969; Yen, 1981) are variations of $\chi_{\mathrm{B}}^{2}$. For example, the statistic implemented by Yen (1981), named $Q_{1}$, uses the mean $\theta$ within each category to obtain the predicted proportions, rather then the median. Furthermore, Yen's index specifies 10 categories, whereas no fixed number is specified with $\chi_{\mathrm{B}}^{2}$. Yen also compared several $\chi^{2}$ fit indices using monte carlo data. An important result of her research was the determination that the $Q_{1}$ statistic was approximately distributed as $\chi^{2}$ with the number of categories minus the number of parameters as the degrees of freedom. Because $\chi_{\mathrm{B}}^{2}$ is similar to the $Q_{1}$ index, these important distributional results should transfer to the former statistic.

McKinley and Mills (1985) studied the performance of several $\chi^{2}$ fit indices in monte carlo research. They found it difficult to choose between the competing methods. In other words, no particular $\chi^{2}$ fit index was clearly superior to others. However, in their research $\chi_{\mathrm{B}}^{2}$ yielded the smallest number of erroneous acceptances of fit under conditions of simulated model-data misfit.

\section{The Purpose and Calculation of Person Fit}

The purpose of a person-fit statistic is to identify examinees whose response patterns are incongruent with the specified response model. Several specific measurement disturbances have been associated with item response aberrancy. For example, Wright (1977) identified response patterns consistent with the hypotheses of "sleeping" and "guessing." Person-fit statistics can further be 
used to identify response patterns associated with specific educational deficits (Tatsuoka, 1982, 1984) or to extract deviant response patterns to create a more nearly unidimensional data matrix (Tatsuoka \& Tatsuoka, 1982). Person-fit statistics also have potential value as response consistency/validity indices in personality measurement (Reise \& Waller, 1990).

Of special interest in this research was a person-fit statistic originally proposed by Levine and Rubin (1979) and standardized by Drasgow, Levine, and Williams (1985). Consider the likelihood function that is to be maximized to estimate $\theta$ :

$$
\begin{aligned}
L \mid \theta & =\sum_{k=1}^{K}\left\{U_{k}\left[\ln P_{k}(\theta)\right]+\left(1-U_{k}\right)\right. \\
& \left.\times\left[\ln Q_{k}(\theta)\right]\right\},
\end{aligned}
$$

where $U_{k}$ is the 0,1 item response,

$P_{k}(\theta)$ is the probability of a correct response given $\theta$,

$Q_{k}(\theta)=1-P_{k}(\theta)$, and

$K$ is the number of items.

The $L$ value represents the height of the likelihood function given the item responses and the item parameters; its first derivative is 0 at the maximum likelihood $\theta$ estimate. $L$ is a simple and direct measure of how well a response pattern conforms to the model. High $L$ indicates good fit, whereas relatively low $L$ indicates poor fit. However, the magnitude of $L$ will depend on $\theta$ level, and the range of $L$ will depend on test length. These problems can be circumvented because for each cell in the 0,1 persons $\times$ items matrix, a standardization can be performed:

$Z_{3}=\frac{\mathrm{L}(\theta)-\mathrm{E}[\mathrm{L}(\theta)]}{\mathrm{SD}[\mathrm{L}(\theta)]}$,

where $E(\theta)$ is the conditional mean (i.e., expected value) and $\operatorname{SD}(\theta)$ is the conditional standard deviation of the likelihood whose formulas are stated in Drasgow et al. (1985, p. 71). The Drasgow $Z_{3}$ index has heretofore been applied only to study person fit (called "appropriateness measurement" by Drasgow et al.). However, their statistical procedure is equally applicable to studying item-fit issues.
To compute $Z_{3}$ for items or examinees, the three terms shown in Equation 3 must be computed for each cell in the examinees $\times$ items response matrix. Then the three terms in Equation 3 must be summed either down a column (for an item) or across a row (for an examinee) of a data matrix. The $Z_{3}$ statistic should be distributed approximately $N(0,1)$ when the data fit the model, and observed values of $Z_{3}$ should be independent of $\theta$ when calculated for examinees, and with item difficulty when calculated for items.

Negative values of $Z_{3}$ when calculated for examinees are associated with response inconsistency (i.e., response patterns that are unlikely, given the measurement model and the $\theta$ estimate). Positive $Z_{3}$ values indicate that the examinee's response pattern was more consistent than the probabilistic IRT model expected, and 0 is the expectation. For items, a negative $Z_{3}$ value indicates an unlikely item response vector, given the estimated item parameters. Positive values of $Z_{3}$ for items indicate response vectors that are more orderly than the probabilistic model and the item parameters predict.

Hence, an advantage of the Drasgow et al. (1985) fit statistic is that two types of misfit can be identified: (1) response vectors that are inconsistent with the model (negative $Z_{3}$ values), and (2) response vectors that are more consistent than the model predicts (positive $Z_{3}$ values). Evaluating misfit with the $Z_{3}$ index thus requires twotailed tests of fit using the normal distribution. By contrast, the $\chi^{2}$ test is a one-tailed fit statistic and does not make a distinction between under- and overfitting an IRT response model.

$\chi_{B}^{2}$ can also be used to assess the degree to which examinees respond according to the specified model. Using $\chi_{\mathrm{B}}^{2}$ to examine person fit is similar to conducting a person response curve (PRC) analysis (Lumsden, 1978; Trabin \& Weiss, 1979). A PRC is a function with item difficulty on the abscissa and the probability of item response on the ordinate. This function can be determined for each examinee and then compared to the expected PRC based on the IRT model. 
If the model fits, the two curves should be close. One method to formally test the degree to which an observed PRC conforms to its expected shape is to compute $\chi_{\mathrm{B}}^{2}$ by interchanging the roles of $j$ and $i$ in Equation 1. In $\chi_{\mathrm{B}}^{2}$ for examinees, $j$ is the interval created by grouping items on difficulty and $i$ is the examinee.

If the observed PRC is different from the expected PRC, this discrepancy should translate into a large and significant $\chi^{2}$. Wright (1977) and Trabin and Weiss (1979) proposed $\chi^{2}$ procedures to study person fit.

\section{Problems With Fit Indices}

Two problems are characteristic of $\chi^{2}$ fit measures. The first problem concerns the intervals: How should the intervals be created, and how many should be constructed? The resulting value of the $\chi^{2}$ statistic will likely be dependent on the decisions made. Second, the number of observations has a strong influence on the $\chi^{2}$ statistic. With sufficiently large sample sizes or numbers of items, it can be shown that $\chi^{2}$ values will tend toward significance (indicating misfit) for items or examinees, respectively. Therefore, with $\chi^{2}$, any IRT model will be rejected if enough data are collected.

Person-fit statistics, in general, are confounded by trait level (Harnisch \& Linn, 1981, p. 140). Although conceptually it makes sense that examinees with very high or very low $\theta$ estimates have less response aberrancy potential than middle-range $\theta$ examinees, strong linear or nonlinear associations between person-fit statistics and $\theta$ estimates (or between item-fit statistics and item parameter estimates) vitiate the index. A second problem is that researchers seldom report the null distributions for their proposed person-fit indices. This leaves the user in a predicament when deciding how to interpret the statistic in practice. A final problem is the general nature of person-fit indices: A significant index does not indicate its cause. Note, however, that $\chi^{2}$ fit indices provide no more or less diagnostic information.

The problems with the $\chi^{2}$ statistic seem less correctable than those of person-fit statistics. Thus, if a person-fit index performs well for both items and examinees, the problems with $\chi^{2}$ can be circumvented by eliminating its use. Before such a proposal can be made, empirical data must be generated comparing the two methods. Hence, $\chi_{\mathrm{B}}^{2}$ was compared with $Z_{3}$ in data simulated to fit the three-parameter logistic IRT model.

\section{Method}

\section{Simulated Datasets}

Eight 50 item $\times 1,000$ examinee 0,1 response matrices were generated under the threeparameter logistic model. The true $\theta$ generating parameters were distributed $N(0,1)$. The true item generating parameters and the resulting internal consistency (coefficient $\alpha$ ) statistics are displayed in Table 1. Internal consistency and model-data fit are distinct concepts, yet coefficient $\alpha$ is shown in Table 1 to gauge the quality of the simulated tests. Datasets 1 through 4 were created with the intention of simulating tests with guessing parameters expected from five-option multiplechoice items, a reasonable spread of item difficulty, and low to moderate internal consistency. Datasets 5 through 8 were generated to have higher internal consistency.

Table 1

Item Discrimination $(a)$, Item Difficulty $(b)$, Guessing Parameter (c), and Coefficient Alpha for the Eight Simulated Datasets

\begin{tabular}{cccccc}
\hline \hline & & \multicolumn{2}{c}{$b$} & & \\
\cline { 3 - 4 } Dataset & $a$ & Shape & Range & $c$ & Alpha \\
\hline 1 & .75 & Uniform & $-2.5,2.5$ & .20 & .75 \\
2 & 1.00 & Uniform & $-2.5,2.5$ & .20 & .78 \\
3 & 1.25 & Uniform & $-2.5,2.5$ & .20 & .83 \\
4 & 1.50 & Uniform & $-2.5,2.5$ & .20 & .84 \\
5 & .75 & Uniform & $-1.5,1.5$ & .10 & .82 \\
6 & 1.00 & Uniform & $-1.5,1.5$ & .10 & .87 \\
7 & 1.25 & Uniform & $-1.5,1.5$ & .10 & .91 \\
8 & 1.50 & Uniform & $-1.5,1.5$ & .10 & .92 \\
\hline
\end{tabular}

\section{Procedure}

To compute $\chi_{\mathrm{B}}^{2}$ for items, examinees were grouped into $10 \theta$ intervals with 100 examinees 
per interval. Ten intervals were selected because this is a common number used in the item-fit literature (e.g., Yen, 1981). This resulted in 7 degrees of freedom. To compute $\chi_{B}^{2}$ for examinees, the item difficulties were grouped into five intervals, resulting in 10 observations (items) per interval. Five intervals were selected in order to have at least 10 observations per interval on which to base the observed proportions. Fewer intervals might have been used, but this would have resulted in a loss of degrees of freedom. With five intervals the statistic had 4 degrees of freedom.

The $Z_{3}$ index was calculated by adding the terms in Equation 3-down columns for items, or across rows for examinees. It was assumed that $Z_{3}$ would be distributed approximately as $\mathrm{N}(0,1)$ and thus the $Z$ distribution is the appropriate null distribution. Previous research (Drasgow et al., 1985) demonstrated that $Z_{3}$ will be approximately normally distributed. All statistical tests of fit involving $Z_{3}$ were two-tailed in order to identify both types of misfit (i.e., under- and overfitting). The risk involved here in using the $Z$ distribution was assumed to be no greater than the risk involved in assuming that $\chi_{\mathrm{B}}^{2}$ is distributed as $\chi^{2}$ when the model is true.

Four analyses were conducted:

1. For each simulated data matrix the proportions of examinees and items identified as not fitting (Type I errors) under several $\alpha$ levels were determined. The selected $\alpha$ levels were $.001, .01$, and .05 .

2. The linear correlations between the fit indices and $\theta$ for examinees, and between the fit indices and the item difficulty for items, were calculated for each dataset. No strong correlations would be expected between these variables.

3. Correlations between $\chi_{B}^{2}$ values and the squared $Z_{3}$ values were computed for examinees and items within each data matrix. (Note that $Z_{3}$ must be squared before being compared to $\chi^{2}$.) Agreement coefficients were also computed by counting the number of cases in which the $Z_{3}$ and $\chi_{\mathrm{B}}^{2}$ fit indices led to the same fit-to-model decisions when both fit indices were evaluated at the same $\alpha$ level.

4. It was determined how well each index performed in identifying misfit when the test model was incorrect (Type II errors). To accomplish this, $\chi_{B}^{2}$ and $Z_{3}$ were computed for examinees and items in Datasets 1 through 3 using the true item parameters of Dataset 4. Furthermore, $\chi_{B}^{2}$ and $Z_{3}$ were computed for examinees and items in Datasets 5 through 7 using the true item parameters from Dataset 8 . With such manipulations, the tests for fit were conducted with an incorrect model, because the parameters were incorrect. In this case, the tests of fit were conducted with true item discrimination parameters that were always higher than the item discrimination parameters used to generate the data. The extent of misfit imposed by these manipulations was one of degree; the more the item parameters used to test fit deviated from the data-generating parameters, the greater was the imposed misfit.

Although many types of misfitting data can be conceived and the performance of each index under different types of response aberrancy can be compared (Drasgow, 1982; Gafni, 1987), this was not the focus of the present research. Rather, the interest was in a general type of misfit, namely when the item discriminations used to test fit were larger than the item discriminations that generated the data. It is important to note that testing fit using incorrect item parameter values forces the examinees' response patterns to be erroneous as well. If a response pattern was determined to fit the model when fit was tested under a correct and incorrect IRT model, then the fit indices would not be considered very sensitive to the values of the item discrimination parameters used to test fit.

\section{Results}

\section{Aberrancy Detection in Data Simulated to Fit the Model}

The proportions of examinees identified by the 
Table 2

Proportion of Examinees and Items Identified as Aberrant Across the Eight Datasets at Critical Values (CV) for Specified Type I Error Rates, for $Z_{3}$ and $\chi_{\mathrm{B}}^{2}$ Statistics

\begin{tabular}{|c|c|c|c|c|c|c|}
\hline & & $Z_{3}$ & & & $\chi_{B}^{2}$ & \\
\hline Examinees & & & & & & \\
\hline $\mathrm{CV}$ & \pm 3.29 & \pm 2.57 & \pm 1.96 & 18.46 & 13.27 & 9.48 \\
\hline Error rate & .001 & .01 & .05 & .001 & .01 & .05 \\
\hline Dataset & & & & & & \\
\hline 1 & .003 & .015 & .039 & .005 & .017 & .081 \\
\hline 2 & .002 & .012 & .041 & .005 & .027 & .108 \\
\hline 3 & .002 & .012 & .042 & .014 & .028 & .096 \\
\hline 4 & .004 & .015 & .053 & .010 & .041 & .109 \\
\hline 5 & .000 & .009 & .054 & .003 & .016 & .090 \\
\hline 6 & .003 & .016 & .047 & .007 & .029 & .091 \\
\hline 7 & .000 & .007 & .049 & .002 & .018 & .096 \\
\hline 8 & .001 & .006 & .036 & .007 & .025 & .094 \\
\hline Total & .001 & .011 & .045 & .006 & .025 & .095 \\
\hline Items & & & & & & \\
\hline $\mathrm{CV}$ & \pm 3.29 & \pm 2.57 & \pm 1.96 & 24.32 & 18.47 & 14.07 \\
\hline Error rate & .001 & .01 & .05 & .001 & .01 & .05 \\
\hline Dataset & & & & & & \\
\hline 1 & .000 & .020 & .060 & .000 & .060 & .200 \\
\hline 2 & .000 & .000 & .020 & .000 & .060 & .160 \\
\hline 3 & .000 & .000 & .040 & .000 & .080 & .220 \\
\hline 4 & .000 & .000 & .040 & .000 & .060 & .200 \\
\hline 5 & .000 & .000 & .080 & .060 & .100 & .280 \\
\hline 6 & .000 & .020 & .040 & .000 & .020 & .200 \\
\hline 7 & .000 & .000 & .040 & .000 & .040 & .200 \\
\hline 8 & .000 & .000 & .060 & .000 & .060 & .220 \\
\hline Total & .000 & .005 & .047 & .007 & .060 & .210 \\
\hline
\end{tabular}

fit indices as misfitting are displayed in the top portion of Table 2. The total proportions across the eight datasets reveal that for examinees, $Z_{3}$ rejected at a rate closer to the specified $Z_{\alpha}$ than $\chi_{\mathrm{B}}^{2} . Z_{3}$ rejected at $.001, .011$, and .045 for $\alpha$ levels $.001, .01$, and .05 . In contrast, $\chi_{B}^{2}$ rejected at $.006, .025$, and .095 for the same three $\alpha$ levels. To a small degree, then, $\chi_{B}^{2}$ overidentified examinees as misfitting under conditions of data simulated to fit the model.

Table 2 also displays the proportions of items identified as aberrant by each index under conditions of data simulated to fit the model. Similar to the results for examinees, $\chi_{B}^{2}$ rejected at a higher rate than $Z_{3}$. Specifically, $\chi_{B}^{2}$ rejected at a .06 rate when $\alpha=.01$, and at a .21 rate when $\alpha=.05 . Z_{3}$ appeared to reject at close to the specified $\alpha$ level for items. The $\chi_{B}^{2}$ rejection rates were in agreement with the findings of McKinley and Mills (1985), who reported that $\chi_{B}^{2}$, when computed to assess item fit, rejected at $.05, .04$, and .07 when fit was evaluated at $\alpha=.01$ under simulations with 1,000 examinees.

Neither index appeared to be confounded by trait level or item difficulty, and the two fit indices were only moderately linearly correlated. In terms of the (mis)fit indications, the two indices behaved similarly for examinees and items in most instances.

\section{Relations Between the Indices}

Several correlations of interest are displayed in Table 3. The first four columns in Table 3 show the linear correlations between the fit indices and the true parameters. The first two columns indicate that there was essentially no 
Table 3

Correlations Between $Z_{3}, \chi_{\mathrm{B}}^{2}, \theta$, and $b$, and Percentage of Fit Decision Agreements

\begin{tabular}{|c|c|c|c|c|c|c|c|c|}
\hline \multirow{4}{*}{$\begin{array}{c}\text { Data- } \\
\text { set }\end{array}$} & \multicolumn{8}{|c|}{ Correlations } \\
\hline & & & \multirow{2}{*}{\multicolumn{2}{|c|}{$b$}} & \multirow{3}{*}{$\begin{array}{l}Z_{3}^{2} \text { vs } \\
\text { Exam- } \\
\text { inees }\end{array}$} & $\chi_{\mathrm{B}}^{2}$ & $\begin{array}{r}\text { Per } \\
\text { Agre }\end{array}$ & $\begin{array}{l}\text { nt } \\
\text { nent }\end{array}$ \\
\hline & \multicolumn{2}{|c|}{$\theta$} & & & & \multirow[b]{2}{*}{ Items } & \multirow{2}{*}{$\begin{array}{l}\text { Exam- } \\
\text { inees }\end{array}$} & \multirow[b]{2}{*}{ Items } \\
\hline & $Z_{3}$ & $\chi_{\mathrm{B}}^{2}$ & $Z_{3}$ & $\chi_{B}^{2}$ & & & & \\
\hline 1 & .03 & .00 & .15 & -.08 & .49 & .34 & 98 & 92 \\
\hline 2 & -.02 & -.04 & .00 & -.05 & .50 & .22 & 98 & 94 \\
\hline 3 & .00 & .04 & .22 & -.03 & .48 & .17 & 97 & 92 \\
\hline 4 & -.03 & .00 & .24 & -.02 & .41 & .33 & 96 & 94 \\
\hline 5 & -.02 & .02 & .13 & .03 & .42 & .11 & 98 & 90 \\
\hline 6 & .04 & .00 & -.19 & .03 & .50 & .05 & 97 & 96 \\
\hline 7 & -.01 & .00 & .25 & .05 & .39 & .17 & 98 & 96 \\
\hline 8 & .05 & .00 & -.06 & .07 & .36 & .23 & 97 & 94 \\
\hline Total & & & & & & & 97 & 94 \\
\hline
\end{tabular}

correlation between examinee fit and $\theta$ for either index. Also, in linear terms, there were no consistent positive or negative trends in the correlational results to warrant rejecting the hypothesis that item fit was uncorrelated with item difficulty. Table 3 further indicates that the two indices were not highly linearly related when calculated for either examinees or items. In a linear sense, then, it appeared that the two indices were functioning differentially.

As indicated in Table 3, across datasets for examinees, the two indices agreed about (mis)fit in 7,806 cases $(97 \%)$ and disagreed in 194 cases (3\%) when both indices were evaluated at $\alpha=.01$. Clearly, the fit decisions made, in a yes/no sense, were quite comparable between the two fit indices. For items across the eight datasets, the two indices made the same (mis)fit decision 374 times $(94 \%)$ and were incongruent only 26 times $(5 \%)$ when fit was evaluated at $\alpha=.01$ for both indices.

Figures $1 \mathrm{a}$ and $\mathrm{lb}$ display the relation between $\theta$ and $Z_{3}$, and between $\theta$ and $\chi_{\mathrm{B}}^{2}$, respectively, for Dataset 1 (comparable results were obtained for the other datasets). No strong relationships are evident in these plots. For both fit indices the variance of fit values seems to decrease at the $\theta$ extremes. Figure lc displays the relation between squared $Z_{3}$ values and $\chi_{\mathrm{B}}^{2}$ values for examinees in
Dataset 1 . Figures $2 \mathrm{a}$ and $2 \mathrm{~b}$ demonstrate that neither $Z_{3}$ nor $\chi_{B}^{2}$ values are related to item difficulty in Dataset 1. Figure $2 \mathrm{c}$ illustrates the relation between squared $Z_{3}$ values and $\chi_{\mathrm{B}}^{2}$ values in the same dataset.

\section{Identifying Simulated Misfit}

Table 4 lists the proportions identified as misfitting for examinees and items, respectively, when the Dataset 4 item parameters were used in place of the true generating item parameters in Datasets 1 through 3, and when the Dataset 8 item parameters were used to test fit in Datasets 5 through 7 . These results show that the proportion of items or examinees identified as misfitting depended on the degree that the generating item parameters deviated from the Dataset 4 or 8 item parameters. Overall, the $\chi_{B}^{2}$ fit index identified more items and examinees as misfitting than did the $Z_{3}$ fit index.

Using either fit index, item misfit was easier to detect than examinee misfit. This may be due to the type of misfit imposed on the data, and to the fact that there were 20 times $(50$ vs. 1,000$)$ the number of observations when calculating item fit as opposed to person fit. The power to detect misfit should improve with an increasing number of observations.

The results in Table 4 clarify two important 
Figure 1

Scatterplots of Fit Indexes for Examinees in Dataset 1

(a) $\theta$ Versus $Z_{3}$

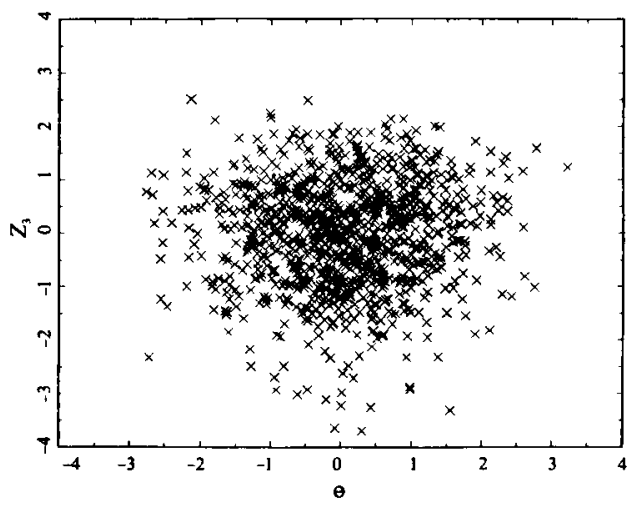

(b) $\theta$ Versus $\chi_{B}^{2}$

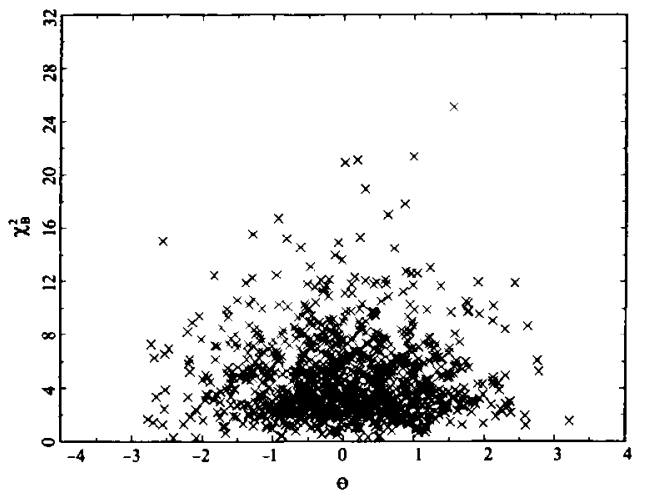

(c) $Z_{3}^{2}$ Versus $\chi_{\mathrm{B}}^{2}$

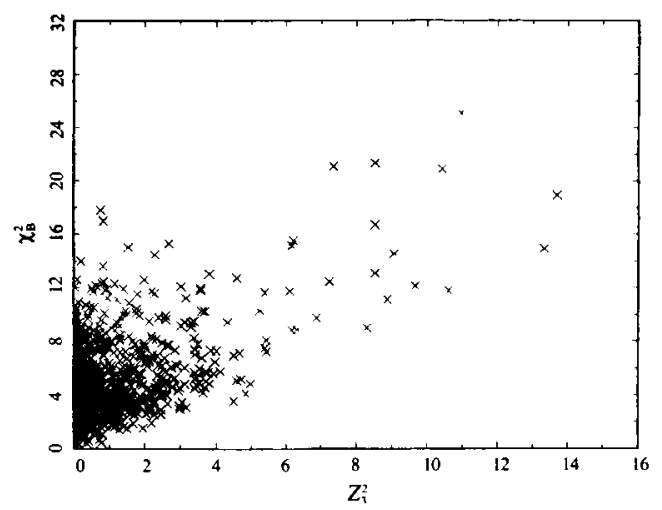

Figure 2

Scatterplots of Fit Indexes for Items in Dataset 1

(a) $b$ Versus $Z_{3}$

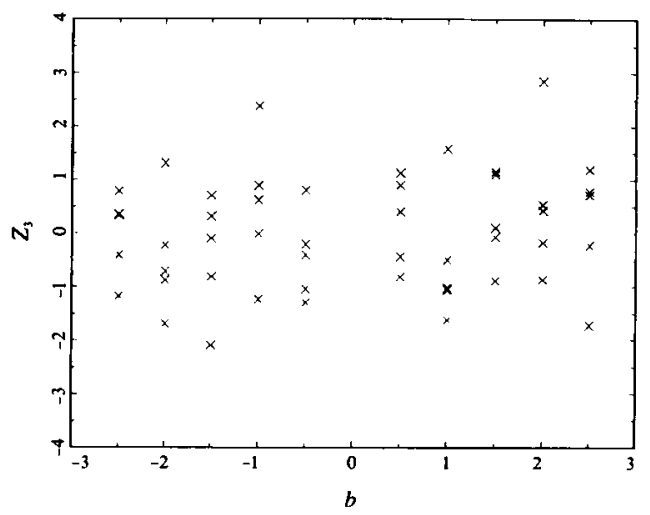

(b) $b$ Versus $\chi_{\mathrm{B}}^{2}$

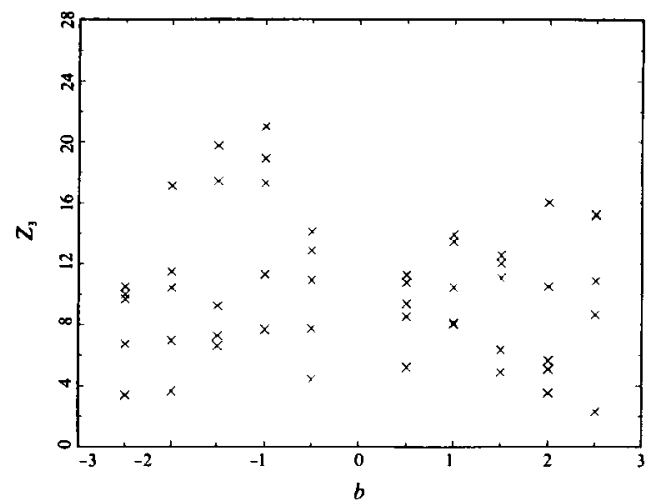

(c) $Z_{3}^{2}$ Versus $\chi_{\mathrm{B}}^{2}$

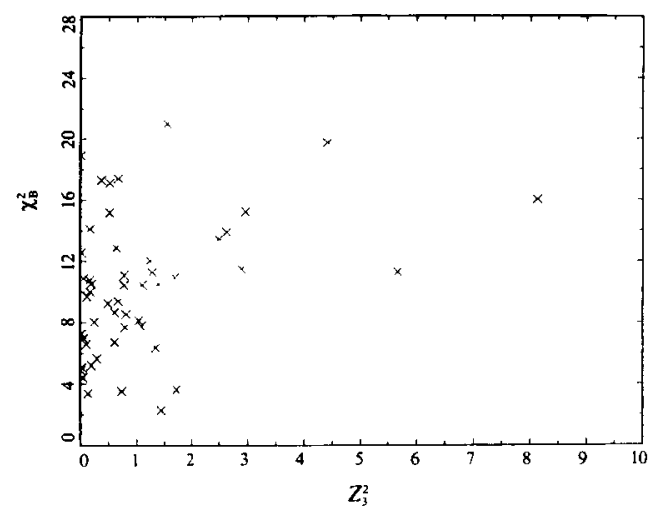


Table 4

Proportion of Examinees and Items Identified as Misfitting When Incorrect Item Parameters Were Used to Test Fit

\begin{tabular}{|c|c|c|c|c|c|c|}
\hline \multirow[b]{2}{*}{ Examinees } & \multicolumn{3}{|c|}{$Z_{3}$} & \multicolumn{3}{|c|}{$\chi_{B}^{2}$} \\
\hline & & & & & & \\
\hline $\mathrm{CV}$ & \pm 3.29 & \pm 2.57 & \pm 1.96 & 18.47 & 13.27 & 9.48 \\
\hline Error rate & .001 & .01 & .05 & .001 & .01 & .05 \\
\hline \multicolumn{7}{|l|}{ Dataset } \\
\hline 1 & .260 & .394 & .548 & .295 & .429 & .575 \\
\hline 2 & .073 & .164 & .276 & .135 & .236 & .361 \\
\hline 3 & .019 & .045 & .104 & .042 & .100 & .188 \\
\hline 5 & .207 & .343 & .496 & .200 & .331 & .521 \\
\hline 6 & .065 & .150 & .281 & .082 & .177 & .314 \\
\hline 7 & .012 & .036 & .098 & .025 & .065 & .161 \\
\hline \multicolumn{7}{|l|}{ Items } \\
\hline $\mathrm{CV}$ & \pm 3.29 & \pm 2.57 & \pm 1.96 & 24.32 & 18.47 & 14.07 \\
\hline Error rate & .001 & .01 & .05 & .001 & .01 & .05 \\
\hline \multicolumn{7}{|l|}{ Dataset } \\
\hline 1 & .980 & 1.000 & 1.000 & .980 & 1.000 & 1.000 \\
\hline 2 & .800 & .920 & .940 & .680 & .840 & .920 \\
\hline 3 & .180 & .280 & .380 & .220 & .400 & .620 \\
\hline 5 & 1.000 & 1.000 & 1.000 & 1.000 & 1.000 & 1.000 \\
\hline 6 & .960 & .980 & 1.000 & .940 & .980 & .980 \\
\hline 7 & . 140 & .400 & .580 & .180 & .360 & .600 \\
\hline
\end{tabular}

issues. First, neither index is sensitive enough to identify small deviations in item discrimination accuracy. Second, when the item discrimination parameters were incorrect, $\chi_{\mathrm{B}}^{2}$ was more sensitive to the misfit that resulted.

\section{Discussion}

The data did not clearly support the use of $Z_{3}$ over $\chi^{2}{ }_{\mathrm{B}}$ or vice versa. $Z_{3}$ held closer to the specified $\alpha$ levels than did $\chi_{B}^{2}$. On the other hand, $\chi_{B}^{2}$ was more sensitive to misfit under the present conditions. However, it appeared that $\chi_{\mathrm{B}}^{2}$ was biased toward rejecting fit. No strong linear relations emerged between the two indices, but the (mis)fit indications they gave, in terms of the percentage of fit decision agreements with respect to examinees and items, were quite comparable. In fact, there were few cases in which one index would indicate misfit and the other would not when the data were simulated to fit the model.

On computational grounds, the $Z_{3}$ index is more "efficient" than the $\chi_{B}^{2}$ index. Also, $Z_{3}$ allows for two types of misfitting to be differentiated: (1) response vectors that are less consis- tent than the model predicts, and (2) response vectors that are too consistent with respect to the specified model. However, researchers have not been overly concerned with this latter type of response aberrancy. $\chi_{\mathrm{B}}^{2}$ requires several critical computation decisions that can affect the results. Furthermore, with $\chi_{B}^{2}$, sample sizes much larger than 1,000 will tend to inflate the test statistic and indicate every item to be misfitting. If used for examinees, long tests will tend to inflate the $\chi^{2}$ statistic. Consequently, it might be appropriate to seek alternative methods to $\chi^{2}$ - both graphical (e.g., Kingston \& Dorans) and statistical (e.g., Drasgow et al., 1985)-to assess fit. The data presented here support the use of $Z_{3}$ to assess fit for items as well as for examinees.

The results demonstrated that $Z_{3}$ was not confounded with $b$ or $\theta$ for items and examinees, respectively. The relation between fit values and the other item parameters could not be calculated because discrimination and guessing were held constant within tests. On the other hand, the same positive results were found with $\chi_{\mathrm{B}}^{2}$. However, other researchers have reported rela- 
tions between $\chi^{2}$ fit and the item parameters. For example, Koch (1983) noted a tendency for large $\chi^{2}$ to result for highly discriminating items.

There are several qualifications pertaining to the results presented here. First, true parameters were used to investigate detection rates. The consequences of using estimated parameters were not addressed. Furthermore, only one type of response aberrancy was addressed in this research, namely the special case when all items in the test are less discriminating for the examinee than for the calibration sample.

It was interesting to observe that the fit indices used in this study appeared to have little difficulty identifying items as misfitting when the model was grossly inappropriate (e.g., testing fit in Dataset 1 using the Dataset 4 item parameters), but were problematic when identifying examinees in the same manner. In many cases, examinees were identified as fitting the model (i.e., having an appropriate response pattern) when fit was computed using the incorrect item discrimination parameter. This result should be considered in applied work.

\section{References}

Andersen, E. B. (1973). A goodness of fit test for the Rasch model. Psychometrika, 38, 123-140.

Bock, R. D. (1972). Estimating item parameters and latent ability when responses are scored in two or more nominal categories. Psychometrika, 37, 29-51.

Drasgow, F. (1982). Choice of test model for appropriateness measurement. Applied Psychological Measurement, 6, 297-308.

Drasgow, F., Levine, M. V., \& Williams, E. A. (1985). Appropriateness measurement with polychotomous item response models and standardized indices. British Journal of Mathematical and Statistical Psychology, 38, 67-86.

Gafni, N. (1987). Detection of systematic and unsystematic aberrance as a function of ability estimate by several person-fit indices. Unpublished doctoral dissertation, University of Minnesota.

Harnisch, D. L., \& Linn, R. L. (1981). Analysis of item response patterns: Questionable test data and dissimilar curriculum practices. Journal of Educational Measurement, 18, 133-146.

Kingston, N. M., \& Dorans, N. J. (1985). The analysis of item-ability regressions: An exploratory IRT model fit tool. Applied Psychological Measurement, 9, 281-288.

Koch, W. R. (1983). Likert scaling using the graded response latent trait model. Applied Psychological Measurement, 7, 15-32.

Levine, M. V., \& Drasgow, F. (1982). Appropriateness measurement: Review, critique, and validating studies. British Journal of Mathematical and Statistical Psychology, 35, 42-56.

Levine, M. V., \& Rubin, D. F. (1979). Measuring the appropriateness of multiple-choice test scores. Journal of Educational Statistics, 4, 269-290.

Lumsden, J. (1978). Tests are perfectly reliable. British Journal of Mathematical and Statistical Psychology, 31, 19-26.

McKinley, R. L., \& Mills, C. N. (1985). A comparison of several goodness-of-fit statistics. Applied Psychological Measurement, 9, 49-57.

Reise, S. P., \& Waller, N. G. (1990). Applications of response pattern aberrancy analysis to personality assessment. Unpublished manuscript.

Tatsuoka, K. K. (1982). A latent trait model for interpreting misconceptions in procedural domains. In D. J. Weiss (Ed.), Proceedings of the 1982 Item Response Theory and Computerized Adaptive Testing Conference (pp. 322-339). Minneapolis: University of Minnesota, Department of Psychology, Psychometric Methods Program.

Tatsuoka, K. K. (1984). Caution indices based on item response theory. Psychometrika, 49, 95-110.

Tatsuoka, K. K., \& Linn, R. L. (1983). Indices for detecting unusual patterns: Links between two general approaches and potential applications. Applied Psychological Measurement, 7, 81-96.

Tatsuoka, K. K., \& Tatsuoka, M. M. (1982). Detection of aberrant response patterns and their effect on dimensionality. Journal of Educational Statistics, 7, 215-231.

Trabin, T. E., \& Weiss, D. J. (1979). The person response curve: Fit of individuals to item characteristic curve models (Research Report 79-7). Minneapolis: University of Minnesota, Department of Psychology, Psychometric Methods Program.

Wright, B. D. (1977). Solving measurement problems with the Rasch model. Journal of Educational Measurement, 14, 97-116.

Wright, B. D., \& Mead, R. J. (1977). BICAL: Calibrating items and scales with the Rasch model (Research Memorandum No. 23). Chicago: University of Chicago, Department of Education, Statistical Laboratory.

Wright, B. D., \& Panchapakesan, N. (1969). A procedure for sample-free item analysis. Educational and Psychological Measurement, 29, 23-48. 
Yen, W. M. (1981). Using simulation results to choose a latent trait model. Applied Psychological Measurement, 5, 245-262.

\section{Author's Address}

Send requests for reprints or further information to Steven P. Reise, Department of Psychology, University of California, Riverside CA 92521, U.S.A. 